Super - sat ur at ed hydr ogen ef f ect s on radi at $i$ on danages in tungst en under the hi gh- fI ux di vert or pl asma i r r adi at i on

\begin{tabular}{|l|l|}
\hline $\begin{array}{l}j \text { our nal or } \\
\text { publ i cat } i \text { on } \mathrm{t} \text { i } \mathrm{tl} \text { e }\end{array}$ & Nucl ear Fusi on \\
\hline vol une & 55 \\
\hline number & 8 \\
\hline page $\mathrm{r}$ ange & 083019 \\
\hline year & $2015-$ 07- 14 \\
\hline URL & ht t p: //hdl . handl e. net /10655/00012796 \\
\hline
\end{tabular}




\title{
Super-Saturated Hydrogen Effects on Radiation Damages in Tungsten under High-Flux Divertor Plasma Irradiation
}

\author{
D. Kato ${ }^{1,2}$, H. Iwakiri ${ }^{3}$, Y. Watanabe ${ }^{4}$, K. Morishita ${ }^{5}$, and T. Muroga ${ }^{1,2}$
}

${ }^{1}$ National Institute for Fusion Science (NIFS), Toki, Gifu 509-5292, Japan

${ }^{2}$ Dept. of Fusion Science, SOKENDAI (The Graduate University for Advanced Studies), Toki, Gifu 509-5292, Japan

${ }^{3}$ University of the Ryukyus, Okinawa 903-0213, Japan

${ }^{4}$ Japan Atomic Energy Agency (JAEA), Rokkasho, Aomori 039-3212, Japan

${ }^{5}$ Institute of Advanced Energy (IAE), Kyoto University, Uji, Kyoto 611-0011, Japan

E-mail contact of main author: kato.daiji@nifs.ac.jp

\begin{abstract}
Tungsten is a prime candidate as the divertor material of the ITER and DEMO reactors, which would be exposed to unprecedentedly high-flux plasmas as well as neutrons. For a better characterization of radiation damages in the tungsten under the divertor condition, we examine influences of super-saturated hydrogen on vacancies in the tungsten. The present calculations based on density functional theories (DFT) reveal unusual phenomena predicted at super-saturated hydrogen concentration: 1) Strongly enhanced vacancy concentration with the super-saturated hydrogen concentration is predicted by a thermodynamics model assuming multiplehydrogen trapping, i.e., hydrogen clusters formation, in the vacancies; and 2) DFT molecular dynamics revealed that hydrogen clusters can prevent a vacancy from recombining with the neighboring crowdion-type selfinterstitial-atom. This suggests that neutron damage effects will be increased in the presence of the hydrogen clusters.
\end{abstract}

\section{Introduction}

Since tungsten will be used for plasma facing component materials in ITER and is a candidate material also for DEMO reactors, tritium retention in neutron damaged tungsten is a key issue. As beryllium co-deposition will dominate tritium inventory in the ITER Be/W wall [1, 2], the radiation damage will play more significant roles for full-W DEMO reactors. Recently, 
experimental works with fission neutrons [3] and high-energy heavy surrogate ions [4, 5] have been performed to evaluate the radiation effects. In these experiments, tungsten specimens with various damage levels upto $0.8 \mathrm{dpa}$ (displacement-per-atom) were exposed to D plasmas with fluxes ranging from $2 \times 10^{18} \mathrm{~m}^{-2} \mathrm{~s}^{-1}$ [3] to $10^{24} \mathrm{~m}^{-2} \mathrm{~s}^{-1}$ [4] and fluences of $6 \times 10^{22}$ $\mathrm{m}^{-2}[3]$ and $10^{26} \mathrm{~m}^{-2}[4,5]$. These works show significant enhancement of the retention in the damaged tungsten and primary roles of radiation induced vacancies and/or vacancy clusters (voids). In the damaged tungsten specimens, D trap concentrations are saturated at $1.0-1.4$ at\% around $0.3 \mathrm{dpa}$, while the damage level at the end of the ITER lifetime would reach to a value as high as 0.6 dpa at the divertor. Roth $[6,7]$ predicted even lower values for the trap concentrations, because spontaneous annihilation of the defects will be enhanced at high temperatures of the divertor. It is noted that we need to take outgassing after the plasma exposures into account in addition to the long-term tritium retention evaluated based on these experimental results. Recently, some modeling works are also undertaken to investigate roles of vacancies in the retention dynamics under simultaneous irradiation of hydrogen isotopes and neutrons, e.g., [8]. However, it is not straightforward to extrapolate the results for ITER and DEMO.

In steady-state divertor plasmas, the tungsten target would be exposed simultaneously to lowenergy $(0.1-100 \mathrm{eV})$ and high-flux $\left(10^{24} \mathrm{~m}^{-2} \mathrm{~s}^{-1}\right)$ plasma particles as well as neutron. Strong enhancement of the hydrogen retention in single- and polycrystalline tungsten specimens has been observed at higher fluxes [9]. The enhanced retention is associated with surface blistering. However, since such low-energy hydrogen bombardment cannot make appreciable displacement (e.g., Frenkel pair) of the tungsten lattice, mechanisms of the blistering are not unravelled. A distinct feature of plasma irradiation from gas permeation obeying the Sieverts' law is that incident hydrogen atoms/ions from the plasma can readily overcome permeation barriers of the surface without high pressures. One can, therefore, assume that under the continuous high-flux implantation local concentration of hydrogen atoms can exceed the solubility limit of the tungsten. In such cases, the tungsten matrix may contain super-saturated hydrogen atoms and sustain extremely high stresses. Therefore, the blistering of tungsten surfaces may be induced by the exceedingly high local concentration of hydrogen atoms beyond the solubility limit of the tungsten [9-11]. Impurities seeded into the divertor plasmas will also play a role in enhancing the local concentrations of hydrogen, because the impurities intruded into tungsten will inhibit hydrogen transport and act as a trapping site of hydrogen. 
In the present paper, for a better characterization of radiation damages in the tungsten under the extreme condition of the divertor materials, we theoretically examine influences of the super-saturated hydrogen on vacancies in the tungsten based on density functional theories.

\section{Theoretical Methods}

In this section, theoretical methods for investigation of vacancy-hydrogen interactions in metals and super-saturated hydrogen effects on the vacancy are described.

\subsection{DFT calculation of vacancy-hydrogen complex in tungsten}

The present DFT calculations were performed with VASP (Vienna Ab-initio Simulation Package) code [12, 13] based on the density functional theories (DFT). In the DFT method, mutually interacting many-electron Schrödinger equations are approximated by a set of independent-particle equations (Kohn-Sham equation) including energy functional of exchange and correlation among electrons. For the exchange-correlation energy functional in the DFT, Perdew-Burke-Ernzerhof (PBE) version of the generalized gradient approximation (GGA) [14] was chosen in the present calculations, because the GGA should give a better approximation to the energy functional in cases that the local electron density changes rapidly, e.g., vacancy and void. Six valence electrons (6s and 5d) assigned to each tungsten atom and one electron of each hydrogen atom are explicitly treated in a field of residual ions, while core electrons of each tungsten atom are represented by a generalized pseudo-potential of the projector augmented wave method [13]. The electron wave functions are expanded in terms of plane-wave basis.

In the present study, two reference bcc super-cells containing 54 and 128 tungsten atoms, respectively, are used with the periodic boundary condition. The Monkhorst-Pack scheme [15] was adopted for k-point sampling in the Brillouin-zone. For the super-cell with 54 tungsten atoms, $6 \times 6 \times 6 \mathrm{k}$-point grids were chosen, and for that with 128 tungsten atoms, $4 \times 4 \times 4$ k-point grids were chosen. The large cut-off energy, i.e., $350 \mathrm{eV}$, was used for the plane-wave basis expansion.

Ionic configurations were relaxed by using a combination of the quasi-Newton method and conjugate-gradient method to the equilibrium configuration until the force of every atom in the super-cell becomes smaller than $0.02 \mathrm{eV} / \AA \AA$. Equilibrium volumes and bulk moduli as well as the total energy are determined by fitting computed energy-volume curves to Birch- 
Murnaghan's equation of state. The present calculations of the lattice constant and the bulk modulus for the tungsten crystal are $3.17 \AA$ and 304 GPa, respectively. For examples, changes in the equilibrium lattice constant of the super cell and the total energy for $\mathrm{W}_{53} \mathrm{~V}_{1} \mathrm{H}_{6}$ are about $+0.02 \AA$ and $-17.07 \mathrm{eV}$, respectively, with respect to those for $\mathrm{W}_{53}$ in the perfect crystal.

The formation energy of a complex of $m$ vacancies and $j$ hydrogen atoms is defined in terms of the total energy of the perfect crystal containing $n$ tungsten atoms, $E\left(\mathrm{~W}_{n}\right)$, that of the super-cell containing $n-m$ tungsten atoms and the complex, $E\left(\mathrm{~W}_{n-m} \mathrm{~V}_{m} \mathrm{H}_{j}\right)$, and that of the hydrogen molecule, $E\left(\mathrm{H}_{2}\right)$, as,

$$
e_{\mathrm{f}}\left(\mathrm{V}_{m} \mathrm{H}_{j}\right)=E\left(\mathrm{~W}_{n-m} \mathrm{~V}_{m} \mathrm{H}_{j}\right)-\frac{n-m}{n} E\left(\mathrm{~W}_{n}\right)-\frac{j}{2} E\left(\mathrm{H}_{2}\right)
$$

Binding energies of each hydrogen atom and the vacancy in the complex are given by, respectively,

$$
\begin{aligned}
& e_{\mathrm{b}}(\mathrm{H})=e_{\mathrm{f}}\left(\mathrm{V}_{m} \mathrm{H}_{j-1}\right)+e_{\mathrm{f}}(\mathrm{H})-e_{\mathrm{f}}\left(\mathrm{V}_{m} \mathrm{H}_{j}\right) \\
& e_{\mathrm{b}}(\mathrm{V})=e_{\mathrm{f}}\left(\mathrm{V}_{m-1} \mathrm{H}_{j}\right)+e_{\mathrm{f}}(\mathrm{V})-e_{\mathrm{f}}\left(\mathrm{V}_{m} \mathrm{H}_{j}\right)
\end{aligned}
$$

where $e_{\mathrm{f}}(\mathrm{H})$ stands for the formation energy of a hydrogen atom at the tetrahedral interstitial site and $e_{\mathrm{f}}(\mathrm{V})$ the mono-vacancy formation energy in the tungsten. Zero-point energy corrections to the binding energies were calculated from differences between the zeropoint energies of the hydrogen atoms in the complex and those of the interstitial hydrogen atoms. For $\mathrm{VH}$ and $\mathrm{VH}_{2}$, the zero-point energies are about $0.1 \mathrm{eV} / \mathrm{H}$. Its values increase significantly for larger complexes due to mutual repulsion among hydrogen atoms, e.g. 0.15 $\mathrm{eV} / \mathrm{H}$ for $\mathrm{VH}_{3}$. Since the zero-point energy of the interstitial $\mathrm{H}$ is $0.27 \mathrm{eV}$, the zero-point energy corrections to the binding energies are obtained to be about $+0.17 \mathrm{eV}$ for $\mathrm{VH}$ and $\mathrm{VH}_{2}$, and only $+0.02 \mathrm{eV}$ for $\mathrm{VH}_{3}$, respectively (see Table 1 ).

\subsection{Statistical thermodynamics model of super-saturated hydrogen effects on vacancies in tungsten}

A statistical thermodynamics model originally proposed by Fukai et al. [16] was used with an extension in order to calculate fractional abundances of vacancy-hydrogen complexes separately for different configurations. Complete description about the present statistical model will be given elsewhere [17]. The similar statistical model has been proposed for formation of the vacancy-hydrogen complexes in alpha iron [18]. In the present model, an 
equilibrium equation for a fractional abundance of the vacancy-hydrogen complex $x_{j} \equiv N_{\mathrm{vH}_{j}} / N_{\mathrm{W}}$ in a tungsten bcc matrix consisting of $N_{\mathrm{W}}$ tungsten atoms at a given temperature $T$ and a given fractional abundance of atomic hydrogen $x_{\mathrm{H}} \equiv N_{\mathrm{H}} / N_{\mathrm{W}}$ is obtained assuming $x_{j}<<1$, as,

$$
\begin{aligned}
& x_{j}=\left(\frac{x_{\mathrm{H}}-x_{\mathrm{t}}}{6}\right)^{j} \omega_{j} \exp \left[-\left(e_{j}-j \times e_{\mathrm{i}}\right) / k T\right], \\
& x_{\mathrm{t}}=\sum_{j} j \times x_{j}
\end{aligned}
$$

where $j$ is the number of hydrogen atoms in the complex, $x_{t}$ a fractional abundance of hydrogen atoms assigned to the complex, and $e_{j}$ and $e_{\mathrm{i}}$ formation energies of the complex $\mathrm{VH}_{j}$ and the interstitial hydrogen atom in the tungsten, respectively. $\omega_{j}$ are statistical weights of the complex containing $j$ hydrogen atoms, and 6 in the denominator of the preexponential factor is the number of the tetrahedral (interstitial) sites per tungsten atom in the bcc unit cell. Eq. (3) is a nonlinear system. Its solutions are, therefore, searched by means of the quasi-Newton method with the initial guess given by,

$$
x_{j}^{(0)}=\left(\frac{x_{\mathrm{H}}}{6}\right)^{j} \omega_{j} \exp \left[-\left(e_{j}-j \times e_{\mathrm{i}}\right) / k T\right] .
$$

\section{Results and discussion}

\subsection{Multiple hydrogen trapping by single mono-vacancy in tungsten}

The multiple hydrogen trapping by the single mono-vacancy in metals has been investigated in pioneering works by Myers et al. [19]. It was assumed that the hydrogen atoms share six octahedral sites in the mono-vacancy (see Fig. 1). Recently, Kato et al. [20] revisited the same issue for tungsten in the context of hydrogen retention in ITER using the DFT methods. Table 1 shows the DFT results [20-22] for the binding energies of hydrogen atoms in the complex $\mathrm{VH}_{j}$. In the table, the results for iron are also shown for comparison. The binding energies in tungsten are remarkably larger than those in iron, primarily due to higher formation energies of interstitial hydrogen atoms (at the tetrahedral sites) in tungsten. DFT calculations give the formation energies of $0.34 \mathrm{eV}$ [23] and $1.07 \mathrm{eV}$ (present calculations) in iron and tungsten, respectively. All of the DFT results give fairly large binding energies indicating that the 
multiple trapping is possible in the tungsten. The binding energy for the $\mathrm{VH}_{4}$ complex of the symmetric configuration (4* in Fig. 1) calculated by Kato [20] is significantly smaller than that of the non-symmetric configuration (4) [21, 22]. There are quite a bit experimental works about the trap energies of $\mathrm{D}$ in $\mathrm{D}^{+}$-irradiated tungsten specimens. Since most of them are based on analysis of thermal desorption spectra of the $\mathrm{D}^{+}$-irradiated specimens, characterization of the $\mathrm{D}$ trap is rather ambiguous. Experimental binding energies in the table are deduced from detrapping energies of $\mathrm{H}$ from $\mathrm{InV}_{2}$ complexes in tungsten and desorption energies of $\mathrm{D}$ from a $\mathrm{D}^{+}$-irradiated single crystal tungsten by subtracting the hydrogen migration energy in tungsten $(0.39 \mathrm{eV}$ [24]). The $\mathrm{H}$ detrapping was measured by means of the gamma-ray perturbed angular correlation technique using the isotope ${ }^{111}$ In which gives a well characterized and reproducible $\mathrm{H}$ trap in the form of $\mathrm{InV}_{2}$ in tungsten [25]. The D desorption is associated with detrapping from a vacancy in the $\mathrm{D}^{+}$-irradiated tungsten from transport analysis of the thermal desorption spectra [26]. The experimental values for the second binding energies are significantly smaller than those for the first one, while the DFT results give almost identical binding energies for both of the first and the second hydrogen atoms in the vacancy. Therefore, as pointed out in $[25,26]$ also, the experimental values for the second binding energy should be associated with the third and latter hydrogen atoms trapped in the vacancy. The present DFT results are in good agreement with the experimental results for the binding energy of $\mathrm{H}$. The binding energies of $\mathrm{D}$ are systematically smaller than those of $\mathrm{H}$ which might be due to isotopic effects. It is noted that there is still an issue regarding the maximum number of hydrogen atoms accommodated in the single vacancy. Liu [27] and Ohsawa [22] have suggested 10 or 12 hydrogen atoms can be accommodated in the single mono-vacancy. It is found that with increasing occupation numbers, the trapping sites are shifted toward the tetrahedral sites [22].

In steady state, number densities of hydrogen atoms at tungsten surfaces exposed to a hydrogen flux $\phi_{\text {in }}$ is given by $C_{\mathrm{s}}=\sqrt{f \phi_{\text {in }} / k_{\mathrm{r}}}$, where $f$ is naively given by one minus the reflection coefficient and $k_{\mathrm{r}}$ recombination coefficients [28]. It is noted that the given equation of the $C_{\mathrm{s}}$ is valid only at the surface provided the diffusion into the bulk is neglected. Fig. 2 shows fractional abundances of the hydrogen atoms, i.e., $x_{\mathrm{H}}=N_{\mathrm{H}} / N_{\mathrm{W}}$, calculated assuming the flux of $10^{24} \mathrm{~m}^{-2} \mathrm{~s}^{-1}$, and using the recombination coefficients reported by Anderl et al. [29]. The fractional abundance for $f=0.1$ is as high as $10^{-6}$ at $670 \mathrm{~K}$. Such 
a high concentration can be compared to that obtained by a high-pressure hydrogen gas exposure of $5 \mathrm{GPa}[30]$.

Fig. 3 shows the hydrogen trapping effect on the mono-vacancy concentration in tungsten calculated by using the present thermodynamics model, Eq. (3). Fractional abundance curves assuming the multiple trapping up to six hydrogen atoms are plotted in the figure. In order to implement calculations, the statistical weight $\omega_{j}$ for each complex $\mathrm{VH}_{j}$ is naively chosen according to the simple octahedral configuration (see Fig. 1). In the present calculation, a fractional abundance of hydrogen atoms is assumed as $10^{-6}$. An extraordinary abundance of the vacancies is predicted in the equilibrium state due to the multiple hydrogen trapping. Temperature dependence is depicted in the figure; at higher temperatures anticipated in the divertor the trapping effects are less but still significant. This is ascribed to strong lowering of the vacancy formation energy by trapping many hydrogen atoms. The formation energies of the larger complexes $\mathrm{VH}_{j}(j \geq 3)$ turn to be smaller than those of the interstitial hydrogen atoms of the same numbers. This results in super abundant vacancies at lower temperatures in the form of the large complexes. At even lower temperatures, the vacancy fractional abundance becomes saturated at the level of the hydrogen fractional abundance, because all hydrogen atoms are segregated in the form of the vacancy-hydrogen complexes, which is depicted by the abundance curves for $\mathrm{VH}_{1-5,6}$ in the figure.

\subsection{Meta-stable state of 111-crowdion with VH6 complex}

Vacancies created by neutrons will readily be recombined with adjacent self-interstitial-atoms (SIAs) leaving smaller numbers of vacancies remaining [31], because recombination of such a vacancy-SIA pair takes place without any activation barrier. The most stable SIA in tungsten is predicted by DFT calculations to be of 111-crowdion configuration [32]. A mono-vacancy and a SIA in the 111-crowdion are automatically recombined, if the vacancy falls into a recombination radius around the major radius of the crowdion which has been determined to be $5.4 \AA$ for tungsten by using a bond-order type inter-atomic potential [33]. The equilibrium atomic configuration of the single 111-crowdion is shown in Fig. 4. One SIA is inserted into an array of lattice atoms in the [111] direction resulting in an extended distortion of the lattice configuration primarily along the [111] direction. The reference super-cell with 128 tungsten atoms was used in the present calculation. Fig. 5 shows a field of atomic displacements in the

string of the crowdion, $\left(z_{n+1}-z_{n}-a\right) / a$, where $z_{n}$ is the generalized coordinate of atom $n$ 
in the string and $a$ the distance between undisturbed lattice atoms along the [111] direction. The field of atomic displacements is approximated by soliton solutions of the sine-Gordon equation in the single-string Frenkel-Kontorova model [34], i.e.,

$$
\begin{aligned}
u_{n} & \equiv z_{n}-n a \\
& \approx \frac{2 a}{\pi} \arctan \left[\exp \left(-\frac{n-n_{0}}{N}\right)\right], \quad u_{n}<<N a
\end{aligned}
$$

Extension of the displacement field is characterized by a dimension-less width, i.e., $N$, which is determined to be 1.8 by fitting the present atomic displacements to the soliton solution. The present width is in good agreement with other DFT results [35]. The formation energy of the 111-crowdion by the present DFT calculation is $9.69 \mathrm{eV}$ which is slightly larger than the value $(9.551 \mathrm{eV})$ reported in $[32,35]$, but within the uncertainty of an experimental value: $9.06 \pm 0.63 \mathrm{eV}$ [36].

The recombination of the SIA in the central string of the 111-crowdion and a vacancy introduced in the adjacent 111-string is confirmed by means of DFT molecular static calculations. Fig. 6 shows a result of the present DFT molecular static calculation; the SIA and the vacancy are automatically recombined recovering the perfect crystal as the equilibrium configuration. It is noted that the image of configurational relaxation (Fig. 6 (b)) is not necessarily on the minimum energy path. The configurational relaxation was performed by using the conjugate-gradient method.

Then, we investigated the recombination in a case that the vacancy is occupied by an octahedron of six hydrogen atoms forming the $\mathrm{VH}_{6}$ complex. The present analysis reveals for the first time that there is a meta-stable state of the 111-crowdion with the $\mathrm{VH}_{6}$ complex inside the recombination radius, as shown in Fig. 7. The figure shows that the octahedron hydrogen cluster prevents the vacancy from recombining with the SIA in the neighbouring 111-crowdion. The string of the crowdion is distorted significantly at the vicinity of the $\mathrm{VH}_{6}$ complex. The octahedron structure of the hydrogen cluster is also deformed by interaction with the crowdion. The formation energy of the meta-stable state is calculated as,

$$
e_{\mathrm{f}}\left(\mathrm{VH}_{6}+\mathrm{SIA}\right)=E\left(\mathrm{~W}_{n-1} \mathrm{VH}_{6}+\mathrm{SIA}\right)-E\left(\mathrm{~W}_{n}\right)-3 \times E\left(\mathrm{H}_{2}\right)
$$

The present result is $12.1 \mathrm{eV}$ which is $0.6 \mathrm{eV}$ smaller than sum of the individual formation energies for the $\mathrm{VH}_{6}$ complex and the 111-crowdion. This indicates that the $\mathrm{VH}_{6}$ complexes can trap the 111-crowdion. Stability of the metastable state at elevated temperatures should be investigated in future studies. This dramatic effect of the hydrogen cluster, which is hitherto 
unknown, implies that the radiation damage remaining after collision cascades by energetic primary knock-on atoms will be increased in the presence of the hydrogen clusters.

\section{Conclusions}

In the review by Roth et al. [6], the radiation damage effects on tritium retention is investigated for the case of full-W ITER option. The neutron-induced hydrogen trap concentrations in tungsten have been assumed to be saturated at most $1 \%$, although the expected irradiation damage in the divertor at the end of the ITER lifetime would reach to a value as high as 0.6 dpa. However, influence of ambient hydrogen atoms on the radiation damage, which is anticipated in the divertor, has never been investigated thoroughly.

The present work based on DFT calculations predicts an enhancement of the radiation damages in the tungsten divertor with super-saturated hydrogen, because 1) multiple hydrogen trapping in a vacancy increases the equilibrium concentration of the vacancy, and 2) the hydrogen cluster stacking with a vacancy suppresses annihilation of the vacancy-SIA pair in neutron damaged tungsten. Reasonable agreement with other DFT calculations [21, 22] was obtained for the binding energies of $\mathrm{H}$ in $\mathrm{VH}_{j}$ complexes. It is, therefore, plausible that the saturation level of neutron-induced trap concentrations will become higher under the influence of the super-saturated hydrogen atoms, because the hydrogen clusters in vacancies will act as an inhibitor of the vacancy-SIA recombination.

\section{Acknowledgements}

This work is partly supported by Joint Usage/Research Program on Zero-Emission Energy Research (Institute of Advanced Energy, Kyoto University, ZE26A-8), and NIFS/NINS under the project of Formation of International Scientific Base and Network. A part of this work was carried out using the HELIOS supercomputer system at Computational Simulation Centre of International Fusion Energy Research Centre (IFERC-CSC), Aomori, Japan, under the Broader Approach collaboration between Euratom and Japan, implemented by Fusion for Energy and JAEA.

\section{Reference}

[1] BREZINSEK, S., et al., "Fuel retention studies with the ITER-Like Wall in JET”, Nucl. Fusion 53 (2013) 083023. 
[2] SCHMID, K., et al., "Quantitative modelling of fuel retention in the JET-C and JETILW wall configurations by WallDYN and predictions for ITER”, J. Nucl. Mater. (2014) in press.

[3] HATANO, Y., et al., "Deuterium trapping at defects created with neutron and ion irradiations in tungsten”, Nucl. Fusion 53 (2013) 073006.

[4] 'T HOEN, M.H.J., et al., "Saturation of deuterium retention in self-damaged tungsten exposed to high-flux plasmas”, Nucl. Fusion 52 (2012) 023008.

[5] TYBURSKA, B., et al., "Deuterium retention in self-damaged tungsten”, J. Nucl. Mater. 395 (2009) 150.

[6] ROTH, J., et al., "Recent analysis of key plasma wall interactions issues for ITER”, J. Nucl. Mater. 390-391 (2009) 1.

[7] ROTH, J. and SCHMID, K., "Hydrogen in tungsten as plasma-facing material”, Phys. Scr. T145 (2011) 014031.

[8] NING, R.H., et al., "Modeling D retention in W under D ions and neutrons irradiation”, J. Nucl. Mater. 430 (2012) 20.

[9] ALIMOV, V.Kh., et al., "Deuterium retention in tungsten exposed to low-energy, highflux clean and carbon-seeded deuterium plasmas”, J. Nucl. Mater. 375 (2008) 192.

[10] CONDON, J.B., SCHOBER, T., “Hydrogen bubbles in metals”, J. Nucl. Mater. 207 (1993) 1.

[11] BUZI, L., et al., "Influence of particle flux density and temperature on surface modifications of tungsten and deuterium retention”, J. Nucl. Mater. 455 (2014) 316.

[12] KRESSE, G., FURTHMÜLLER, J., "Efficient iterative schemes for ab initio totalenergy calculations using a plane-wave basis set”, Phys. Rev. B 54 (1996) 11169.

[13] KRESSE, G., JOUBERT, D., "From ultrasoft pseudopotentials to the projector augmented-wave method”, Phys. Rev. B 59 (1999) 1758.

[14] PERDEW, J.P., BURKE, K., ERNZERHOF, M., “Generalized Gradient Approximation made simple”, Phys. Rev. Lett. 77 (1996) 3865.

[15] MONKHORST, H.J., PACK, J.D., “Special points for Brillouin-zone integrations”, Phys. Rev. B 13 (1976) 5188. 
[16] FUKAI, Y., "Formation of superabundant vacancies in $\mathrm{M}-\mathrm{H}$ alloys and some of its consequences: a review”, J. Alloys Comp. 356-357 (2003) 263.

[17] FERNANDEZ, N., FERRO, Y., and KATO, D., "Hydrogen diffusion and vacancies formation in tungsten: Density Functional Theory calculations and statistical models”, accepted for publication in Acta Mater. (2015).

[18] MIRZAEV, D.A., et al., "Formation of hydrogen-vacancy complexes in alpha iron”, Phys. Metals Metallogr. 113 (2012) 923.

[19] MYERS, S.M., et al., "Theoretical examination of the trapping of ion-implanted hydrogen in metals”, Phys. Rev. B 33 (1986) 854.

[20] KATO, D., et al., "First-principle study on binding energy of vacancy-hydrogen cluster in tungsten”, J. Plasma Fusion Res. Ser. 8 (2009) 404.

[21] HEINOLA, K., et al., "Hydrogen interaction with point defects in tungsten”, Phys. Rev. B 82 (2010) 094102.

[22] OHSAWA, K., et al., "Trapping of multiple hydrogen atoms in a tungsten monovacancy from first principles”, Phys. Rev. B 82 (2010) 184117.

[23] TATEYAMA, Y. And OHNO, T., "Stability and clusterization of hydrogen-vacancy complexes in $\alpha$-Fe: An ab initio study”, Phys. Rev. B 67 (2003) 174105.

[24] FRAUENFELDER, R., "Solution and diffusion of hydrogen in tungsten”, J. Vac. Sci. Technol. 6 (1969) 388.

[25] FRANSENS, J.R., et al., "Hydrogen-vacancy interaction in tungsten”, J. Phys.: Condens. Matter 3 (1991) 9871.

[26] POON, M., et al., "Modelling deuterium release during thermal desorption of $\mathrm{D}^{+}$irradiated tungsten”, J. Nucl. Mater. 374 (2008) 390.

[27] LIU, Y.L., et al., "Vacancy trapping mechanism for hydrogen bubble formation in metal”, Phys. Rev. B 79 (2009) 172103.

[28] PENG, H.Y., et al., "Effect of tungsten and carbon surface mixed layer on deuterium permeation in tungsten”, J. Nucl. Mater. 438 (2013) S1063.

[29] ANDERL, R.A., et al., "Deuterium transport and trapping in polycrystalline tungsten”, Fusion Technol. 21 (1992) 745. 
[30] SUGIMOTO, H. and FUKAI, Y., "Solubility of hydrogen in metals under high hydrogen pressure: thermodynamical calculations”, Acta Metall. Mater. 40 (1992) 2327.

[31] PARK, N.-Y., et al., "Molecular dynamics simulation of irradiation damage in tungsten”, Nucl. Instrum. Methods Phys. Res. B 265 (2007) 547.

[32] HGUYEN-MANH, D., et al., "Self-interstitial atom defects in bcc transition metals: Group-specific trends”, Phys. Rev. B 73 (2006) 020101(R).

[33] AHLGREN, T., et al., "Bond-order potential for point and extended defect simulations in tungsten”, J. Appl. Phys. 107 (2010) 033516.

[34] DUDAREV, S.L., “Coherent motion of interstitial defects in a crystalline material”, Phil. Mag. 83 (2003) 3577.

[35] DERLET, P.M., et al., "Multiscale modelling of crowdion and vacancy defects in bodycentered-cubic transition metals”, Phys. Rev. B 76 (2007) 054107.

[36] NEKLYUDOV, I.M., et al., "Interstitial atoms in tungsten: Interaction with free surface and in situ determination of formation energy”, Phys. Rev. B 78 (2008) 115418. 
TABLE 1. DFT results of binding energies $(\mathrm{eV})$ for $\mathrm{H}$ in $\mathrm{VH}_{j}$ comlexes. Present results [20] were obtained using a reference super-cell with 54 atoms. ZPE means the results with zeropoint energy correction. Results of Heinola [21] and Ohsawa [22] include the ZPE correction. Experimental values [25] and [26] are binding energies of $\mathrm{H}$ in $\mathrm{InV}_{2}$ complexes of tungsten and those of $\mathrm{D}$ in mono-vacancies of a $\mathrm{D}^{+}$-irradiated single crystal tungsten, respectively (see text).

\begin{tabular}{|c|c|c|c|c|c|c|c|}
\hline$j$ & $\mathrm{Fe}$ & $\mathrm{W}$ & W ZPE & Heinola[21] & Ohsawa[22] & Exp.[25] & Exp.[26] \\
\hline 1 & 0.64 & 1.22 & 1.39 & 1.43 & 1.318 & $1.16(2)$ & $0.95(3)$ \\
\hline 2 & 0.67 & 1.23 & 1.40 & 1.41 & 1.308 & $0.99(2)$ & $0.68(3)$ \\
\hline 3 & 0.46 & 1.10 & 1.12 & 1.22 & 1.082 & \multirow{4}{*}{$<0.7$} & \multirow{4}{*}{ - } \\
\hline 4 & 0.35 & 0.82 & 0.92 & 1.11 & 1.015 & & \\
\hline 5 & 0.45 & 1.12 & 1.12 & 1 & 0.929 & & \\
\hline 6 & 0.08 & 0.32 & - & 0.47 & 0.677 & & \\
\hline
\end{tabular}




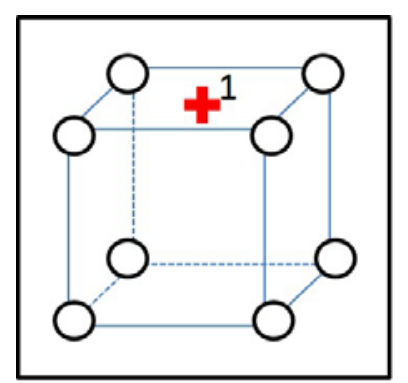

1

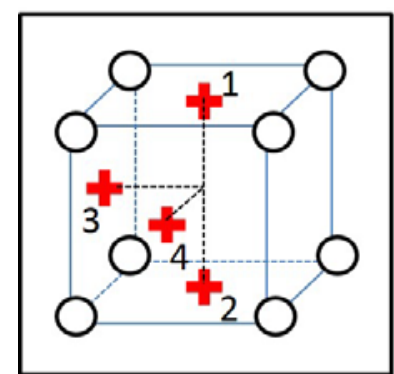

4

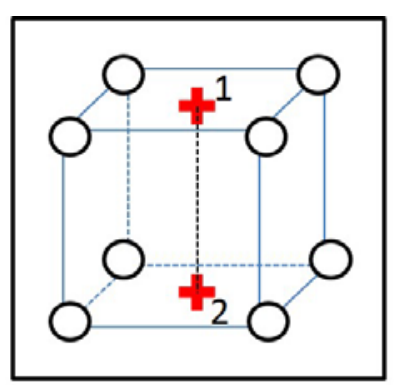

2

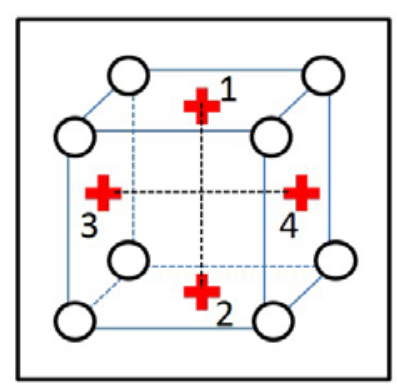

4*

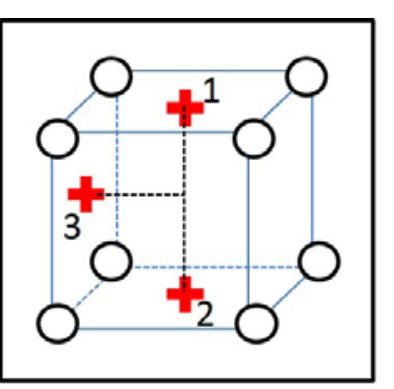

3

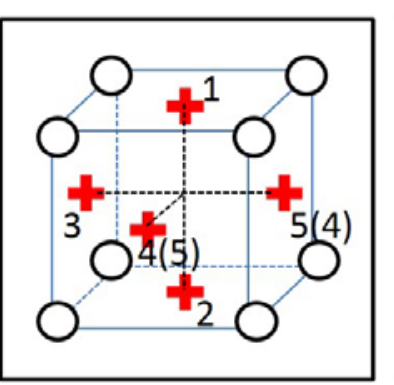

5

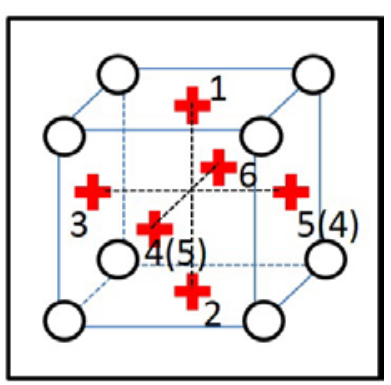

6

FIG. 1. Octahedral configuration of hydrogen atoms (crosses) in mono-vacancy. Two nonequivalent configurations are shown for $\mathrm{VH}_{4}$. Statistical weight of each configuration is $\omega_{j}=6,3,12,12(3), 6,1$ for $\mathrm{j}=1,2,3,4\left(4^{*}\right), 5,6$, respectively.

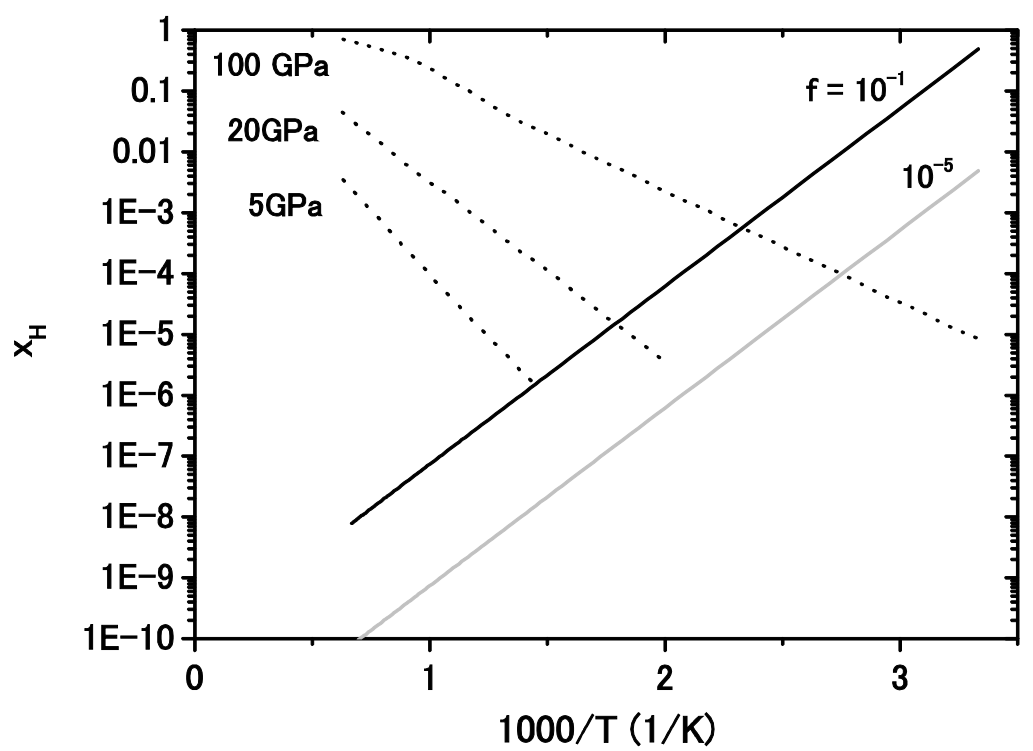

FIG. 2. Hydrogen fractional abundance in tungsten. Solid lines are the fractional abundances in steady state at incident hydrogen ion flux $10^{24} \mathrm{~m}^{-2} \mathrm{~s}^{-1}$ neglecting diffusion into the bulk, and 
dashed lines hydrogen solubility under hydrogen gas $\left(\mathrm{H}_{2}\right)$ exposure calculated by Sugimoto and Fukai [30].

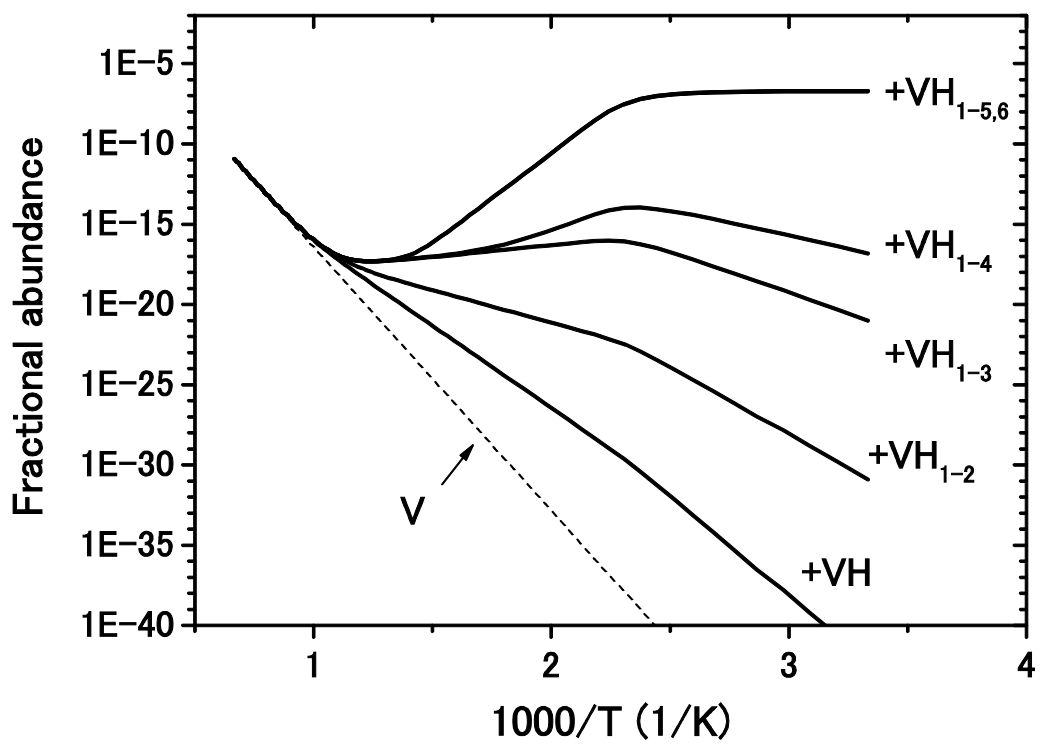

FIG. 3. Equilibrium fractional abundance of $\mathrm{VH}_{\mathrm{j}}$ complexes in tungsten as a function of inverse temperature. A fractional abundance of hydrogen atoms is assumed as $10^{-6}$. The dashed line stands for the thermal vacancy.

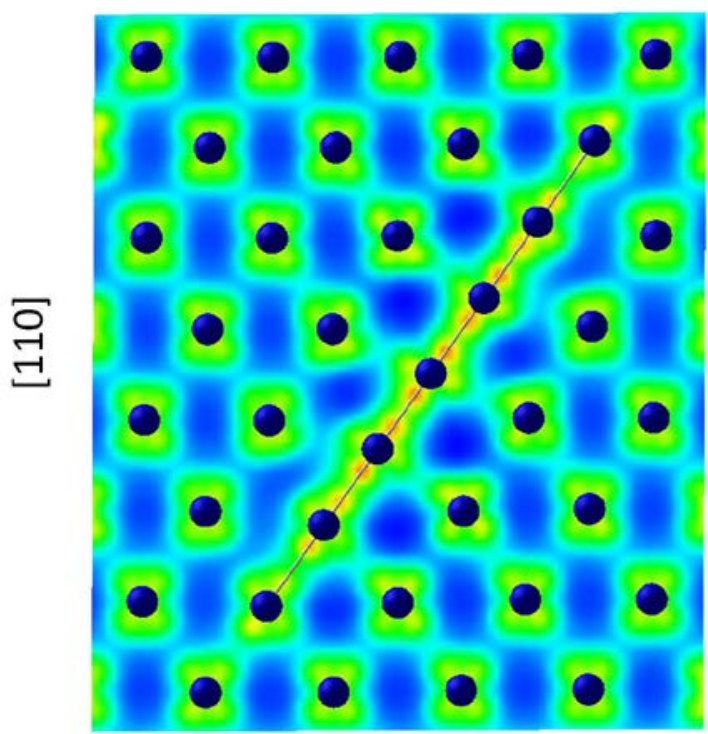

[001]

FIG. 4. Atomic configuration and electron charge density of 111-crowdion. Blue balls are tungsten atoms. The crowdion is indicated by an array of bonded atoms along the [111] 
direction. Colors on the surface indicate electron densities; red indicates higher electron densities while blue indicates depletion of the electron density.

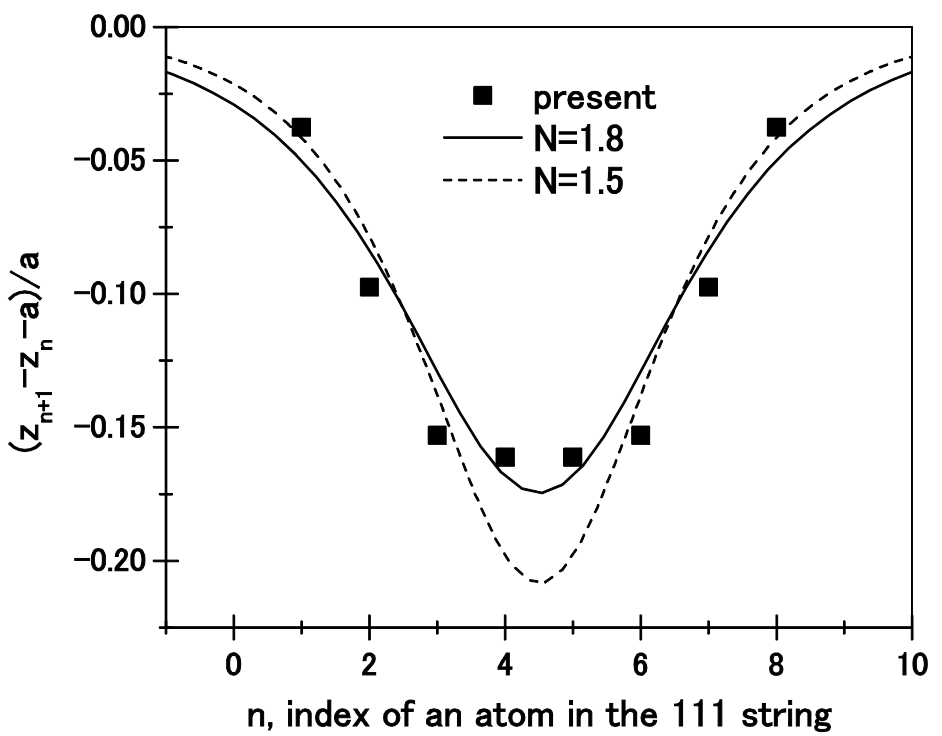

FIG. 5. Field of atomic displacements in the 111-crowdion. Solid squares are present DFT results and curves single-string Frenkel-Kontorova model [Eq.(5)] assuming $n_{0}=5$.
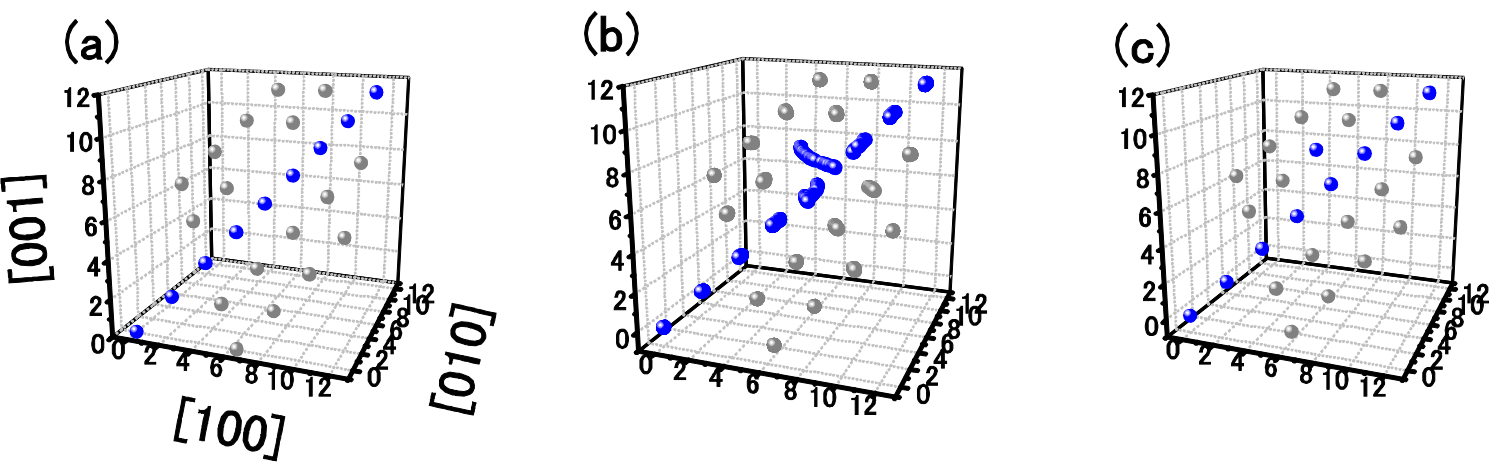

FIG. 6. Atomic configurations for recombining mono-vacancy and 111-crowdion. Blue balls are atoms initially belonging to the central string of the 111-crowdion, light grey atoms in the first- and second-nearest 111-strings on the (110) surface. (a) Initial configuration, (b) image of configurational relaxation by the conjugate-cradient method, and (c) equilibrium (perfect crystal). 


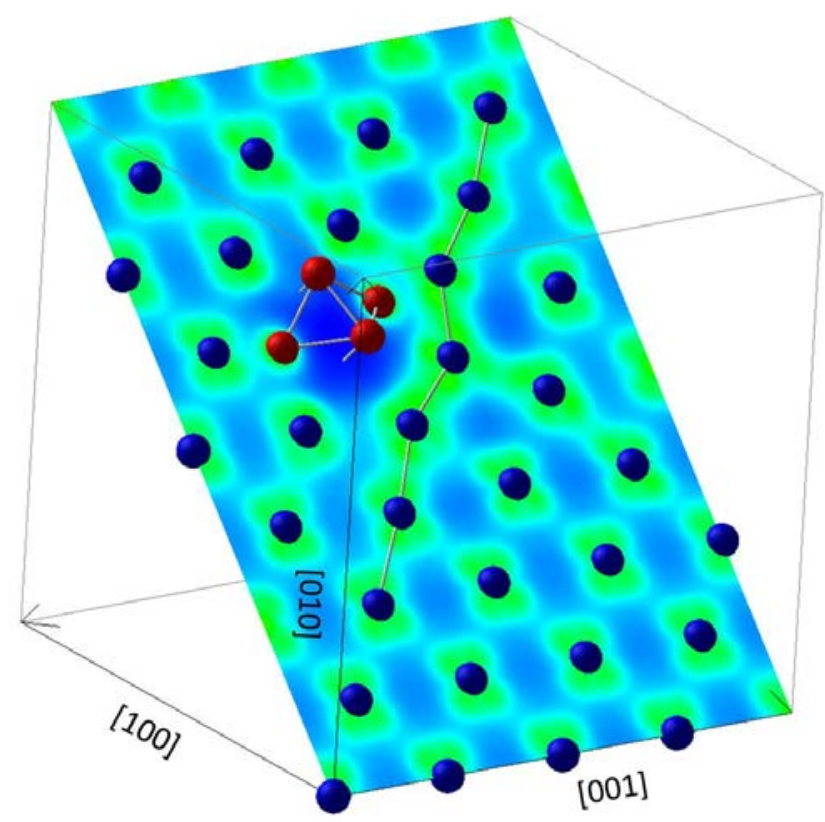

FIG. 7. Octahedron hydrogen cluster stacking with a vacancy $\left(\mathrm{VH}_{6}\right)$ preventing it from recombining with a 111-crowdion SIA in tungsten. Blue balls are tungsten and red balls hydrogen atoms, respectively. Tungsten atoms only on (110) surface are drawn in the figure. Another two hydrogen atoms of $\mathrm{VH}_{6}$ are behind the surface. The crowdion structure distorted by the presence of $\mathrm{VH}_{6}$ is indicated by a connected bond. 